\title{
Multiple regression analysis of perinatal conditions, physical development, and complications in assisted reproduction singletons
}

\author{
Junfeng $\mathrm{Li}^{1}$, Xiuhong $\mathrm{Fu}^{1}$, Jingshang $\mathrm{Lv}^{2}$, Lina Cui ${ }^{2}$, Rongxiang $\mathrm{Li}^{1}$, Aihong Bai ${ }^{1}$, Haoran Wang ${ }^{3}$, \\ Xian Tang ${ }^{4}$
}

${ }^{1}$ Department of Reproductive Medicine, Luohe Central Hospital of Henan Province, Luohe, China; ${ }^{2}$ Department of Obstetrics and Gynecology, Luohe Central Hospital of Henan Province, Luohe, China; ${ }^{3}$ Department of Cardiology, Luohe Central Hospital of Henan Province, Luohe, China; ${ }^{4}$ Department of Reproductive Medicine, Loudi Central Hospital of Hunan Province, Loudi, China

Contributions: (I) Conception and design: J Li, X Fu; (II) Administrative support: J Lv, L Cui; (III) Provision of study materials or patients: J Li, X Fu, J Lv; (IV) Collection and assembly of data: R Li, A Bai; (V) Data analysis and interpretation: J Li, H Wang, X Tang; (VI) Manuscript writing: All authors; (VII) Final approval of the manuscript: All authors.

Correspondence to: Xian Tang. Department of Reproductive Medicine, Loudi Central Hospital of Hunan Province, No. 51 Changqing Road, Loudi, China. Email: ljflijunfeng2021@163.com.

Background: There is no clear clinical conclusion on whether assisted reproductive technology (ART) increases maternal and child risk and affects infant birth and development. This study aimed to perform a multiple regression analysis of the perinatal maternal and infant conditions in an assisted reproductive singleton pregnancy and the physical development and complications of such infants at 6 months old.

Methods: This study enrolled 145 singleton pregnant women who were admitted to Luohe Central Hospital between December 2017 and December 2019 to undergo in vitro fertilization and embryo transfer as a research group, and 160 singleton pregnant women who were naturally conceived at the same time and delivered at our hospital were selected as the control group. The relevant data of the patients were collected, and the perinatal conditions, neonatal complications, physical development and NBN score of infants aged 6 months were compared between the two groups. Multivariate logistic regression was used to analyze risk factors for perinatal complications in assisted reproduction singleton pregnancy.

Results: The incidence of complications in the perinatal period in the study group was significantly higher than that in the control group $(\mathrm{P}<0.05)$. However, there was no significant difference in the incidence of complications such as very low birth weight, respiratory distress, neonatal hemolysis, hyperbilirubinemia, hypoglycemia, infectious pneumonia and neonatal asphyxia between the two groups $(\mathrm{P}>0.05)$. Likewise, at 6 months of age, the infants in the research group showed no significant difference to the control group in physical development indicators, including weight, head circumference, body mass index, height and NBN score $(\mathrm{P}>0.05)$. The analysis of the unconditional multivariate logistic regression model revealed hypertension during pregnancy, placental previa, premature membrane rupture, gestational age $<32$ weeks, and very low birth weight as risk factors that affect perinatal complications in patients with assisted reproductive singleton pregnancy $(\mathrm{P}<0.05)$.

Conclusions: The incidence of complications in perinatal patients with assisted reproductive singleton pregnancy is higher than that of natural singleton pregnancy, but there is no significant difference in physical development, NBN score and complications of 6 months old infants.

Keywords: Assisted reproduction; perinatal period; infant physical development; complications

Submitted Jul 12, 2021. Accepted for publication Sep 15, 2021.

doi: $10.21037 / \mathrm{tp}-21-400$

View this article at: http://dx.doi.org/10.21037/tp-21-400 


\section{Introduction}

According to World Health Organization (WHO) statistics, one in seven couples in the world encounters reproductive health issues (1). A recent infertility research survey in China has found that infertility affects about $10 \%$ of married couples, and there is an upward trend year by year (2).

Assisted reproductive technology (ART) is a clinically assisted medical approach for people facing infertility which includes artificial insemination (AI) and in vitro fertilization and embryo transfer (IVF-ET). IVF-ET technology is widely used and can achieve certain clinical effects (3). Studies have shown that individuals who become pregnant through ART can experience complications, such as placenta previa and placental adhesions, which can affect maternal and infant outcomes and adversely affect the growth and development of the baby after birth (4). However, at present, there is no clear conclusion on whether ART elevates the risk for mothers and their children or affects the birth and development of infants (5).

Therefore, we conducted a study to compare the perinatal maternal and infant conditions between 145 cases of ART singleton pregnancy and 160 cases of naturally conceived singleton pregnancy who were admitted to our hospital, and observed physical development and complications in both groups of infants at 6 months, to provide a reference for clinical diagnosis and treatment. We present the following article in accordance with the STROBE reporting checklist (available at https://dx.doi. org/10.21037/tp-21-400).

\section{Methods}

\section{General information}

The clinical treatment data of 145 singleton pregnant women who received IVF-ET and were admitted to our hospital between December 2017 and December 2019 were selected. These patients (the study group) had an average age of $30.41 \pm 5.18$ years, the average number of pregnancies was $1.41 \pm 0.52$ times, the average number of births was $1.16 \pm 0.41$ times, and the gestational week at delivery was $38.41 \pm 1.42$ weeks. The inclusion criteria were as follows: (I) infertility due to tubal blockage or male semen-related factors; (II) diagnosed as intrauterine singleton pregnancy by imaging examination; (III) no endocrine disease; and (IV) complete clinical data. The exclusion criteria were as follows: (I) patients with underlying diseases such as chronic heart disease, diabetes, and hypertension; (II) immune, chromosomal, or genetic diseases in one or both of the parents; and (III) withdrawal from the study during the research or poor intervention compliance. Additionally, 160 singleton pregnant women who conceived naturally and gave birth in our hospital were selected as the control group. These patients (the control group) had an average age of $30.51 \pm 5.12$ years, the average number of pregnancies was $1.37 \pm 0.44$, the average number of births was $1.21 \pm 0.34$, and the gestational week at delivery was $38.52 \pm 1.16$ weeks.

There was no significant difference in general information between the two groups of patients $(\mathrm{P}>0.05)$, and they were comparable.

The study was approved by medical ethics committee of Luohe Central Hospital (No. 20170526). The patients' family members were aware of and agreed to this study, and signed the relevant informed consent. The study was conducted in accordance with the Declaration of Helsinki (as revised in 2013).

\section{Study methods}

The maternity examination, delivery status, and newborn data of the two groups were collected. The infants were followed up for 6 months, and their physical development and neurobehavioral development was monitored. Neonatal behavioral neurological assessment (NBNA) was used to evaluate neurobehavioral development of the infants. Observation items included 6 items of behavioral ability, 4 items of passive muscle tension, 4 items of active muscle tension, 3 items of primitive reflex and 3 items of general state, a total of 20 items. Each score has three points $(0,1,2)$. Full score 40, $<35$ score abnormal (6).

\section{Perinatal conditions and complications}

Maternal pregnancy/delivery, including gestational age, mode of delivery, premature rupture of membranes, placental abruption, premature birth, fetal distress, threatened abortion, preeclampsia, threatened premature birth, poly or oligohydramnios, restricted fetal growth, stillbirth, severe fetal malformations, etc.

\section{Neonatal complications}

Neonatal complications included very low birth weight, respiratory distress, neonatal hemolysis, hyperbilirubinemia, hypoglycemia, infectious pneumonia and neonatal asphyxia.

\section{Follow-up}

The infants were followed up to the age of 6 months via 
Table 1 Comparison of perinatal conditions between the study and control groups (n, \%)

\begin{tabular}{lccc}
\hline Perinatal conditions & Study group & Control group & $\chi^{2}$ \\
\hline $\mathrm{N}$ & 145 & 160 & - \\
Normal delivery & $51(35.17)$ & $82(51.25)$ & 7.996 \\
Complications & & & \\
Maternal entry into ICU & $6(4.14)$ & $2(1.25)$ \\
Premature delivery & $19(13.10)$ & $8(5.00)$ \\
Cesarean section & $62(42.76)$ & $65(40.63)$ \\
Other complications & $7(4.83)$ & $3(1.87)$ \\
Total & $94(64.83)$ & $78(48.75)$ \\
\hline
\end{tabular}

Table 2 Comparison of neonatal complications between the study and control groups (n, \%)

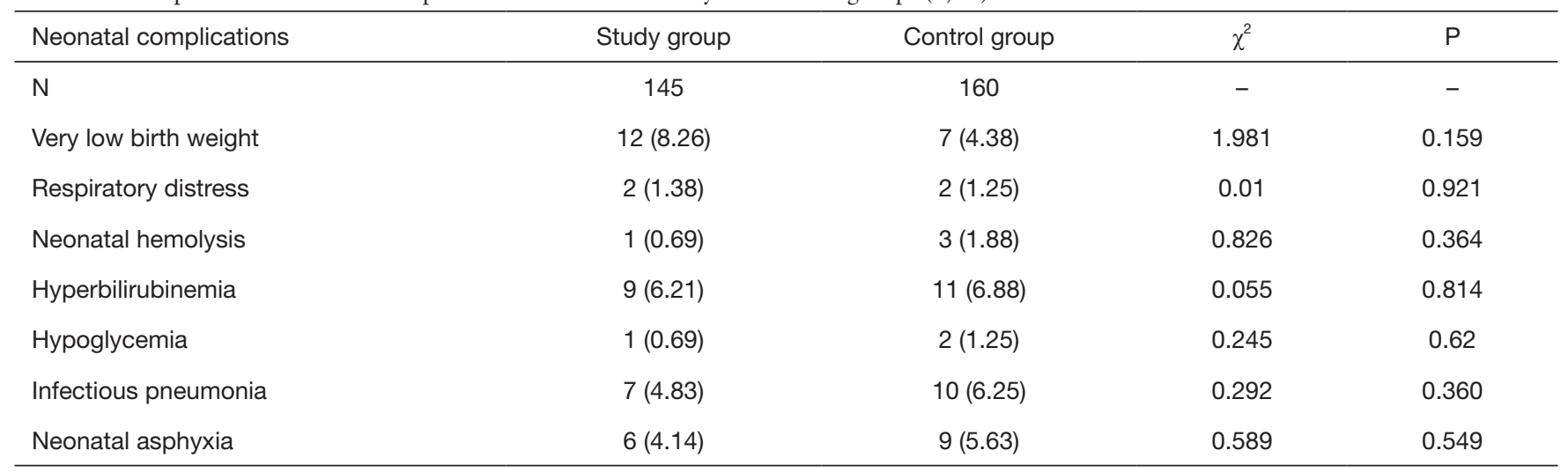

WeChat, telephone, or home visit. The final follow-up was on August 30, 2020. The content of the follow-up included weight, length, head circumference, and body mass index (BMI).

\section{Statistical methods}

The data in this study were statistically analyzed using SPSS22.0 software (SPSS, Chicago, Illinois, USA). The measurement data were described as the mean \pm standard deviation $(\bar{x} \pm s)$ and compared by $t$-test. Count data were described as the pass rate or composition ratio and compared by the $\chi^{2}$ test. Multivariate logistic regression was used to analyze the risk factors for perinatal complications in ART singleton pregnancy. The difference was considered statistically significant with $\mathrm{P}<0.05$.

\section{Results}

Comparison of the perinatal conditions of the two groups

As shown in Table 1, the incidence of perinatal complications was statistically significantly higher in the study group than in the control group $(\mathrm{P}<0.05)$.

\section{Comparison of neonatal complications between the two groups}

As shown in Table 2, there was no significant difference in the incidence of neonatal complications, such as very low birth weight, respiratory distress, neonatal hemolysis, hyperbilirubinemia, hypoglycemia, infectious pneumonia and neonatal asphyxia between the study group and the control group $(\mathrm{P}>0.05)$. 
Table 3 Comparison of the physical development and NBN score of 6-month-old infants between the study and control groups ( $\bar{x} \pm s)$

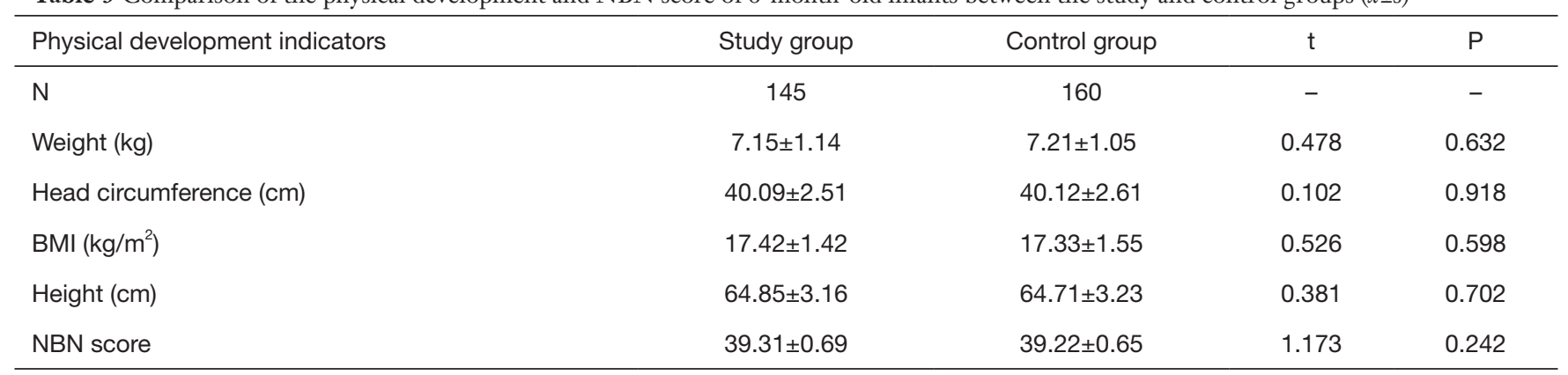

\section{Comparison of the physical development and NBN score of 6-month-old infants between the two groups}

As shown in Table 3, the infants in the study group showed no significant differences in physical development indicators, such as weight, head circumference, BMI, height and NBN score, compared to those in the control group $(\mathrm{P}>0.05)$.

\section{Single-factor analysis of perinatal complications in assisted reproduction singleton pregnancy}

According to the above results, the majority of women in both groups experienced perinatal complications. According to the perinatal conditions described in Table 1, the study participants were divided into groups; those who had a normal delivery were defined as the no complications group, and the rest were regarded as the complications group.

The results of the univariate analysis showed that hypertension during pregnancy, gestational diabetes, placental previa, premature rupture of membranes, gestational age $<32$ weeks, and very low birth weight were factors affecting the risk of perinatal complications in an assisted reproduction singleton pregnancy $(\mathrm{P}<0.05)$ (Table 4).

\section{Analysis of multiple factors affecting perinatal complications in assisted reproduction singleton pregnancy}

After unconditional multivariate logistic regression model analysis, hypertension during pregnancy, placental previa, premature rupture of membranes, gestational age $<32$ weeks, and very low birth weight were found to be independent risk factors for perinatal complications in an assisted reproduction singleton pregnancy $(\mathrm{P}<0.05)$ (Table 5).

\section{Discussion}

With the continuous development of ART, IVFET has become an effective treatment for individuals with infertility. However, the safety of ART and the health risks faced by children born through ART have gradually received clinical attention. Although most children conceived using ART are healthy, individual differences still exist, which has resulted in concern about ART and the health of mothers and babies (7). Therefore, exploration of the factors affecting perinatal and infant health after ART has important clinical value in paving the way for targeted intervention measures and reducing the impact of ART on mothers and infants (8).

To date, a clear conclusion on the impact of ART on perinatal complications and neonatal short- and long-term outcomes has not been reached (9). Studies have shown that after IVF-ET treatment, the risk of complications such as postpartum hemorrhage, placental abruption, and premature rupture of membranes during the perinatal period is higher than that with naturally conceived pregnancies (10). However, it has also been reported that IVF-ET treatment does not increase the risk of perinatal complications but is associated with a higher cesarean section rate (11). Therefore, the present study selected 145 women who underwent IVF-ET treatment in Luohe Central Hospital and 160 women who conceived naturally during the perinatal period. The incidence of complications in the study group was observed to be significantly higher than that in the control group. Logistic regression model analysis showed that hypertension during pregnancy, placental previa, and premature rupture of membranes gestational age $<32$ weeks, and very low birth weight were risk factors for perinatal complications in ART singleton pregnancy. These 
Table 4 Single-factor analysis of perinatal complications in assisted reproduction singleton pregnancy

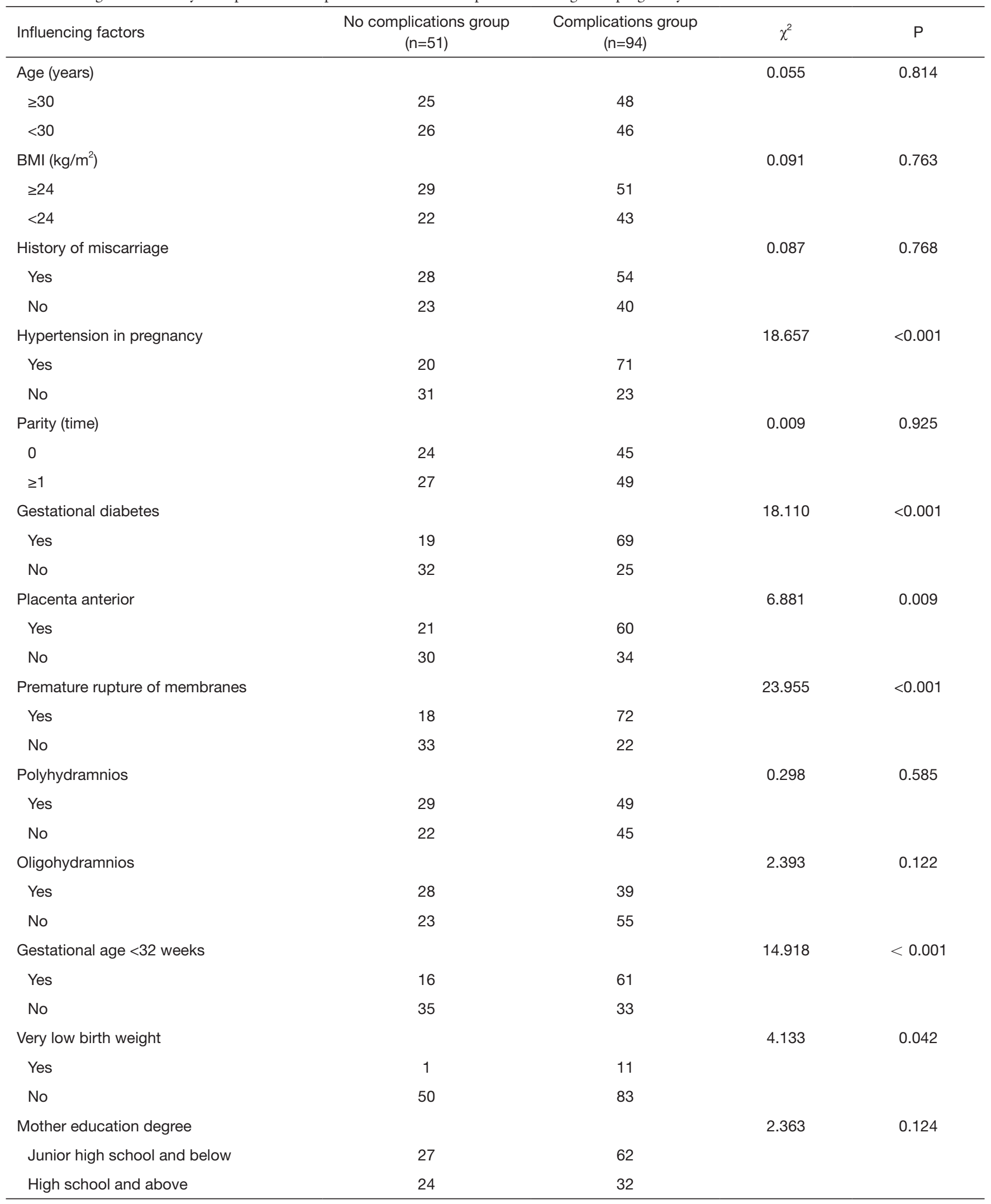


Table 5 Analysis of multiple factors affecting perinatal complications in assisted reproduction singleton pregnancy

\begin{tabular}{lccccc}
\hline Influencing factors & $\begin{array}{c}\text { Regression } \\
\text { coefficients }\end{array}$ & Standard error & Wald $\chi^{2}$ & P & OR (95\% Cl) \\
\hline Hypertension in pregnancy (yes vs. no) & 0.915 & 0.205 & 7.459 & 0.025 & $2.49(1.670-3.731)$ \\
Gestational diabetes (yes vs. no) & 0.506 & 0.316 & 5.184 & 0.201 & $1.65(0.892-3.081)$ \\
Placenta anterior (yes vs. no) & 0.987 & 0.147 & 9.157 & $<0.001$ & $2.68(2.011-3.579)$ \\
Premature rupture of membranes (yes vs. no) & 0.946 & 0.152 & 8.595 & 0.011 & $2.57(1.911-3.469)$ \\
Gestational age <32 weeks (yes vs. no) & 0.977 & 0.231 & 7.112 & 0.032 & $2.656(1.689-4.178)$ \\
Very low birth weight (yes vs. no) & 0.922 & 0.342 & 8.670 & 0.007 & $2.514(1.286-4.915)$ \\
\hline
\end{tabular}

findings are similar to those of previous reports.

The research has indicated that hypertension in pregnancy is related to factors such as age, parity, obesity, multiple pregnancies, diabetes, and chronic immune diseases. Ovulation induction and human chorionic gonadotropin treatment in ART patients during pregnancy may activate the renin-angiotensin-aldosterone system, which is related to the occurrence of pregnancy-induced hypertension $(12,13)$. Furthermore, some studies have shown that high levels of chorionic gonadotropin can damage the blood vessels of the placenta in early pregnancy, leading to placental hypoxia and pregnancy complications (14). However, ART patients may have a history of frequent abortion or intrauterine operations, which can damage the endometrium and cause inflammation, causing endometrial vascular defects. After ART implantation, due to the insufficient blood supply caused by such endometrial vascular defects, the fertilized egg stretches the lower part of the uterus and increases the area of the placenta, resulting in placenta previa (15). Furthermore, Nakamura et al. (16) stated that when some ART patients undergo placental decompression therapy, the fetal reduction can cause amnionitis and fetal membrane inflammation, resulting in fetal membrane dysplasia and leaving them prone to premature rupture of membranes and premature delivery. Therefore, it is necessary to closely monitor the perinatal condition of ART patients, detect potential pregnancy complications as early as possible, and give early intervention and treatment to reduce the risk of perinatal complications in patients and obtain a good pregnancy outcome (17).

Additionally, in this study, neonatal complications among women with ART and natural pregnancies and their infants at 6 months were observed, and no significant difference was found between the two groups. Although previous foreign studies have suggested that ART singleton babies have a higher risk of respiratory distress than non-ART singleton babies, this has yet to be confirmed (18). Scholars such as Lledo et al. (19) have reported that IVF-ET technology affects infants' development. Another study found that children born with the help of IVF-ET experience similar growth and development to children born through natural pregnancy (20). Therefore, in the future, it is necessary to expand the sample size and conduct multi-center, longterm, targeted research to establish whether there is a difference between children conceived with IVF-ET and those conceived naturally. Follow-up of children, both those conceived naturally and with IVF-ET, until adulthood or even beyond is also needed to ensure the authenticity and accuracy of experimental results.

\section{Conclusions}

In summary, complications in the perinatal period are higher in women with ART singleton pregnancies than in natural singleton pregnancies. However, there is no significant difference in the physical development, NBN score and complications of their infants at 6 months of age. Early interventions for complications during the perinatal period in women with ART singleton pregnancies can maximize protection for the mother and child and reduce the occurrence of adverse events.

\section{Acknowledgments}

Funding: None.

\section{Footnote}

Reporting Checklist: The authors have completed the STROBE reporting checklist. Available at https://dx.doi. org/10.21037/tp-21-400 
Data Sharing Statement: Available at https://dx.doi. org/10.21037/tp-21-400

Conflicts of Interest: All authors have completed the ICMJE uniform disclosure form (available at https://dx.doi. org/10.21037/tp-21-400). The authors have no conflicts of interest to declare.

Ethical Statement: The authors are accountable for all aspects of the work in ensuring that questions related to the accuracy or integrity of any part of the work are appropriately investigated and resolved. The study was approved by medical ethics committee of Luohe Central Hospital (No. 20170526). The patients' family members were aware of and agreed to this study, and signed the relevant informed consent. The study was conducted in accordance with the Declaration of Helsinki (as revised in 2013).

Open Access Statement: This is an Open Access article distributed in accordance with the Creative Commons Attribution-NonCommercial-NoDerivs 4.0 International License (CC BY-NC-ND 4.0), which permits the noncommercial replication and distribution of the article with the strict proviso that no changes or edits are made and the original work is properly cited (including links to both the formal publication through the relevant DOI and the license). See: https://creativecommons.org/ licenses/by-nc-nd/4.0/.

\section{References}

1. Tobing VY, Afiyanti Y, Rachmawati IN. Following the cultural norms as an effort to protect the mother and the baby during the perinatal period: An ethnographic study of women's food choices. Enferm Clin 2019;29 Suppl 2:831-6.

2. Romanenko TG, Sulimenko OM. Prevention of preeclampsia in women with multiple pregnancy after assisted reproduction. Wiad Lek 2020;73:494-7.

3. Herrero A, Lluna J, Lucea JA. Bioethical analysis of the impact of Assisted Reproduction Techniques (ART) on the health of children and mothers. Medicina y Ética - AbrilJunio 2020;3:307-25.

4. Li X, Li X, Sun Y, et al. Effect of Y Chromosome Microdeletions on the Pregnancy Outcome of Assisted Reproduction Technology: a Meta-analysis. Reprod Sci 2021. [Epub ahead of print]. doi: 10.1007/s43032-02000387-0.

5. Arnold C, Johnson H, Mahon C, et al. The effects of eating disorders in pregnancy on mother and baby: a review. Psychiatr Danub 2019;31:615-8.

6. Sheridan-Pereira M, Ellison P H, Helgeson V. The Construction of a Scored Neonatal Neurological Examination for Assessment of Neurological Integrity in Full-Term Neonates. Journal of Developmental \& Behavioral Pediatrics 1991;12:25-30..

7. Dolan R, Shaw J, Hann M. Pregnancy in prison, Mother and Baby Unit admission and impacts on perinatal depression and 'quality of life'. Journal of Forensic Psychiatry and Psychology 2019;30:1-19.

8. Simopoulou M, Sfakianoudis K, Giannelou P, et al. Navigating assisted reproduction treatment in the time of COVID-19: concerns and considerations. J Assist Reprod Genet 2020;37:2663-8.

9. Squires EL. Perspectives on the development and incorporation of assisted reproduction in the equine industry. Reprod Fertil Dev 2019;31:1753-7.

10. Páez JCS, Arreseygor VG, Zgrablich P. Obesity and the possibility of conceiving a child during assisted reproduction treatment: An Argentinian experience. JBRA Assist Reprod 2020;24:115-7.

11. Stapleton PA. Assisted Reproduction Policy in Canada: Framing, Federalism, and Failure, by Dave Snow. Publius: The Journal of Federalism 2019;8:1.

12. Wang MJ, Kuper SG, Sims B, et al. Opioid Detoxification in Pregnancy: Systematic Review and Meta-Analysis of Perinatal Outcomes. Am J Perinatol 2019;36:581-7.

13. Hall M, Endress D, Hölbfer S, et al. SARS-CoV-2 in pregnancy: maternal and perinatal outcome data of 34 pregnant women hospitalised between May and October 2020. J Perinat Med 2020;49:138-40.

14. Kosc K. Reproduction on the Reservation: Pregnancy, Childbirth, and Colonialism in the Long Twentieth Century by Brianna Theobald (review). Canadian Journal of History 2020;19:533.

15. Madjunkov M, Dviri M, Librach C. A comprehensive review of the impact of COVID-19 on human reproductive biology, assisted reproduction care and pregnancy: a Canadian perspective. J Ovarian Res 2020;13:140.

16. Nakamura $M$, Oba $T$, Takita $H$, et al. Differences in perinatal outcomes according to forming period of single umbilical cord in singleton pregnancy. Prenat Diagn 2019;39:588-94.

17. Liu KE, Hartman M, Hartman A. Management of thin endometrium in assisted reproduction: a clinical practice guideline from the Canadian Fertility and Andrology Society. Reprod Biomed Online 2019;39:49-62. 
18. Medical Tourism and the Egress of Economic Depression: The Case of Assisted Reproduction Services in Greece. Journal of Economics and Public Finance 2019;5:227.

19. Lledo B, Bernabeu A, Diaz M, et al. Effect of the vaginal microbiome on the pregnancy rate in patients undergoing assisted reproduction techniques. Fertility and Sterility
2019;112:193.

20. Sfontouris I. Assisted reproduction treatment during the Covid-19 pandemic: considerations based on ovarian physiology. Fertility and Sterility 2020,31:662-5.

(English Language Editor: J. Reynolds and J. Chapnick)
Cite this article as: $\mathrm{Li} \mathrm{J,} \mathrm{Fu} \mathrm{X,} \mathrm{Lv} \mathrm{J,} \mathrm{Cui} \mathrm{L,} \mathrm{Li} \mathrm{R,} \mathrm{Bai} \mathrm{A,}$ Wang H, Tang X. Multiple regression analysis of perinatal conditions, physical development, and complications in assisted reproduction singletons. Transl Pediatr 2021;10(9):2347-2354. doi: $10.21037 /$ tp-21-400 